\title{
Invasive Lobular Carcinoma of the Breast: Appearance on Digital Breast Tomosynthesis
}

\author{
Ahuva Grubstein ${ }^{a, b}$ \\ Yael Rapson a,b \\ Sara Morgenstern ${ }^{b, c}$ \\ Itai Gadiela,b \\ Amit Haboosheh ${ }^{d}$ \\ Rinat Yerushalmi ${ }^{b, e}$ \\ Maya Cohen ${ }^{a, b}$
}

a Department of Radiology, Rabin Medical Center, Beilinson Campus, Petah Tikva, Israel;

b Sackler Faculty of Medicine, Tel Aviv University, Tel Aviv, Israel;

' Department of Pathology, Rabin Medical Center, Beilinson Campus, Petah Tikva, Israel;

${ }^{d}$ Department of Radiology, Carmel Medical Center, Haifa, Israel;

e Davidoff Center, Rabin Medical Center, Beilinson Campus, Petah Tikva, Israel

\section{Keywords}

Tomosynthesis · Lobular carcinoma

\section{Summary}

Background: The aim of this study was to characterize the signs of invasive lobular carcinoma of the breast on digital breast tomosynthesis (DBT) imaging. Patients and Methods: The study group included 23 women with pathologically proven invasive lobular carcinoma of the breast for whom both digital mammography (DM) and DBT images were available. The images were read jointly by 2 experienced breast radiologists. Findings were recorded according to the descriptors in the Breast Imaging and Reporting Data System lexicon and correlated with the detailed pathology results. Results: In 21 of the 23 patients, the combination of DM and DBT yielded pathologic findings (91\%). Architectural distortions or spiculations were demonstrated in $87 \%$ of cases. The addition of DBT to DM improved lesion detection by more clearly depicting both the lesion margins and architectural distortions. Only 2 lesions were occult by both DM and DBT, including 1 lesion in a peripheral location that was not incorporated in the standard mediolateral oblique and craniocaudal views. Conclusion: DBT improves the detection of invasive lobular carcinoma lesions by more clearly depicting architectural distortions and spiculations.

(c) 2016 S. Karger GmbH, Freiburg

\section{Introduction}

Invasive lobular carcinoma (ILC) is the second most common type of invasive breast cancer, accounting for $10-15 \%$ of all invasive breast carcinomas [1-3].

The detection of ILC on screening digital mammography (DM) is challenging due to both technical factors related to the methodology and the pathologic features unique to the lesion. DM produces a 2-dimensional image of overlapping breast tissues which poses the risk of obscuring a mass or increased tissue density. Furthermore, as the single-file growth pattern of ILC produces little desmoplastic reaction, with no hemorrhage, necrosis, or calcification, it presents only mild architectural distortions that are difficult to identify on the mammogram [1-3]. The published sensitivity of DM in detecting ILC is only $57-81 \%$. Normal mammographic findings are reported more frequently with ILC (up to $19 \%$ of cases) than with other invasive breast cancers $[1,2,4]$.

Digital breast tomosynthesis (DBT) is an emerging technology which involves the acquisition of multiple low-dose projections of the breast from which cross-sectional images are produced. Total radiation exposure and breast compression are equal to or slightly higher than those of conventional mammography [5]. Several studies have shown that DBT has better capabilities than mammography in lesion detection and characterization. By partially removing the tissue above and below the plane of the lesion, size and margins can be more readily assessed [6-8].

The aim of the present study was to characterize the appearance of ILC on DBT imaging.

\section{Patients and Methods}

Over 1,600 women underwent biopsies in our institution (a tertiary medical center), between 2010 and 2014. Of these, 65 biopsy pathology results were ILC.

\section{KARGER}

() 2016 S. Karger GmbH, Freiburg

Fax +497614520714 
Table 1. Distribution of pathology results in 23 patients with invasive lobular carcinoma

\begin{tabular}{llll}
\hline Pathologic findings & $\begin{array}{l}\text { Initial US-guided } \\
\text { core biopsy } \\
(\mathrm{n}=22)\end{array}$ & $\begin{array}{l}\text { Initial stereotactic } \\
\text { VAB } \\
(\mathrm{n}=1)\end{array}$ & $\begin{array}{l}\text { Final surgical } \\
\text { pathology }^{\mathrm{a}} \\
(\mathrm{n}=6)\end{array}$ \\
\hline Pure ILC & 21 & & 3 \\
ILC and IDC & 1 & 1 & $1^{\mathrm{b}}$ \\
ILC and LCIS & & & $\begin{array}{l}1 \\
\text { ILC and DCIS }\end{array}$ \\
LCIS & & $1^{\mathrm{c}}$ \\
\hline
\end{tabular}

${ }^{\mathrm{a} O n l y}$ few final pathology results were accessible because most patients underwent surgery in external facilities.

${ }^{b}$ Pure ILC on initial biopsy.

'ILC and LCIS on initial VAB. The surgical pathology report stated that the VAB site was visualized, but no residual invasive component was evident.

US = Ultrasound $; \mathrm{VAB}=$ vacuum-assisted biopsy; ILC = invasive lobular carcinoma; IDC = invasive ductal carcinoma; LCIS = lobular carcinoma in situ; DSIC = ductal carcinoma in situ.
Fig. 1. Pathology slide, H\&E stain $\times 20$ magnification: infiltrative lobular carcinoma cells in 'Indian file arrangement' and as single cells are seen in a desmoplastic stroma.

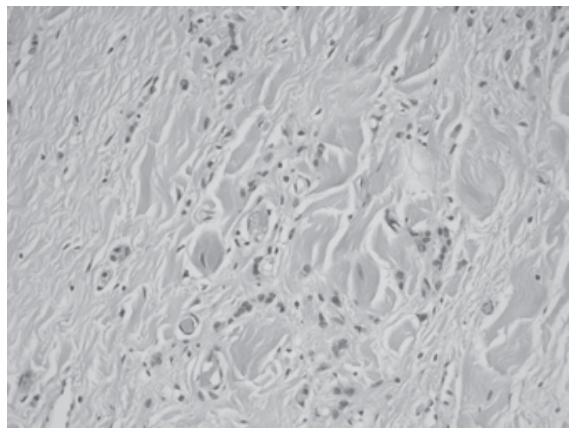

We excluded 42 women for whom we did not have DM and DBT studies from the time of diagnosis prior to the biopsy, leaving us with a study group of 23 women pathologically diagnosed with ILC and for whom both DM and DBT studies were available. All patients also underwent ultrasound studies. In 10 (43.8\%) cases, we had access to magnetic resonance imaging (MRI) studies (some of the studies which were performed in external facilities were not available).

\section{Imaging Techniques}

In all patients, craniocaudal (CC) and mediolateral oblique (MLO) views of each breast were obtained by DM and DBT at the same time (Selenia Dimensions, Hologic, Bedford, MA, USA). Images were interpreted on a dedicated workstation equipped with 2 5-megapixel monitors (SecureView Dx, Hologic). The DM and DBT studies were reviewed in consensus by 2 radiologists with 2-8 years of experience in reading mammograms, and 2-6 years in reading DBT. The readers were aware of the pathology results; reading was aimed at detecting and characterizing the lesion (masses, increased density, architectural distortion, and microcalcifications). Findings were recorded according to the descriptors of the Breast Imaging and Reporting Data System (BI-RADS) lexicon and correlated with the detailed pathology results. Each reader had to subjectively score where the findings were more conspicuous - either on DM or DBT - or whether they were equal. The study was approved by the Institutional Review Board.

\section{Results}

The 23 study patients ranged in age from 44 to 90 years (mean \pm standard deviation, $64 \pm 12$ years). Breast density categories (a-d) were distributed as follows: a - fatty, 2 (9\%) patients; b - scattered fibroglandular, 8 (35\%) patients; c - heterogeneously dense, 8 (35\%) patients; $\mathrm{d}$ - dense, 5 (21\%) patients. There was an even distribution between non-dense and dense BI-RADS categories (simi-

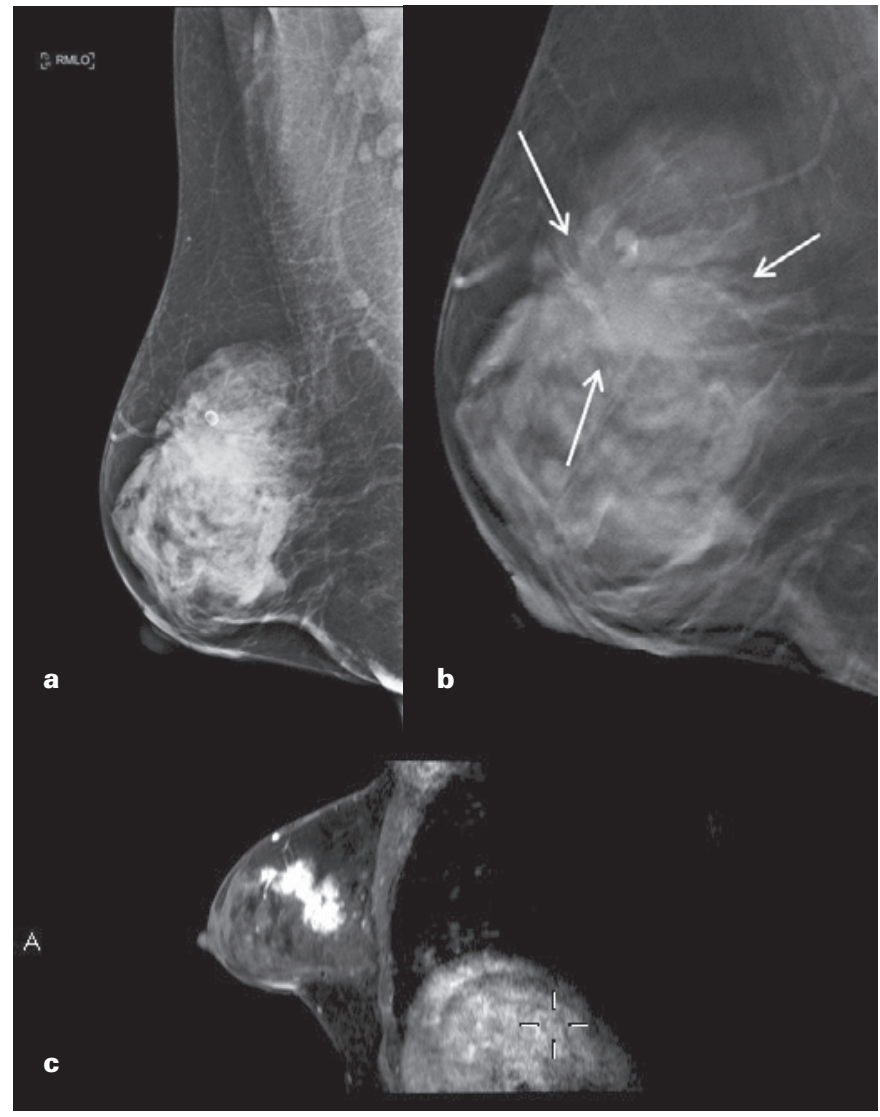

Fig. 2. 62-year-old woman presenting with a right breast palpable mass. a Digital mammography right mediolateral oblique (RMLO) view demonstrates BI-RADS 4 breast density, vague architectural distortion, and a cluster of microcalcifications in the upper quadrant. b Digital breast tomosynthesis (DBT) RMLO demonstrates architectural distortion and a spiculated mass in the upper outer quadrant (arrows). c 3D T1-weighted magnetic resonance imaging after contrast administration, sagittal reconstruction, demonstrates the spiculated mass lesion as well as the distortion, matching the DBT appearance.

lar to the general population). The distribution of the pathology results is shown in table 1. A typical image of ILC in a core biopsy is shown in figure 1.

In 21 (91\%) of the 23 patients, the combination of DM and DBT yielded pathologic findings. Table 2 describes the appearance of the 
Table 2. Lesion characteristics in combined digital mammography (DM) and digital breast tomosynthesis (DBT) studies in 23 patients with invasive lobular carcinoma and differences in lesion conspicuity between modalities

\begin{tabular}{lcccc}
\hline Lesion characteristics & Patients, $\mathrm{n}(\%)$ & \multicolumn{2}{c}{ Conspicuity of finding by modality } \\
\cline { 3 - 5 } & & $\mathrm{DM}$ & $\mathrm{DBT}$ & Equal \\
\hline Architectural distortion & $13(57)$ & & 10 & 3 \\
Mass & $10(43)$ & 1 & 7 & 2 \\
Asymmetric density & $9(39)$ & & 7 & 2 \\
Calcifications $^{\text {a }}$ & $4(17)$ & & 1 & 3 \\
\hline
\end{tabular}

${ }^{\mathrm{a}} 1$ case of a mass with calcifications was more evident on DBT.

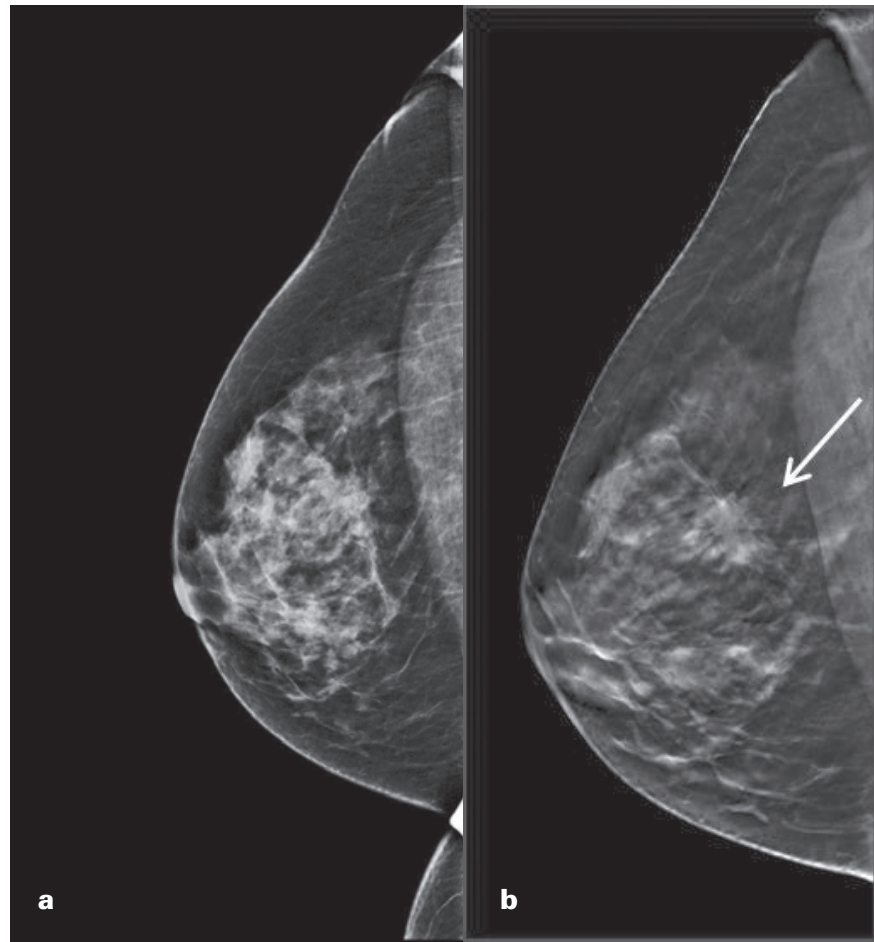

Fig. 3. 67-year-old woman, screening mammogram. a Digital mammography right craniocaudal (RCC) view demonstrates focal asymmetry. b Digital breast tomosynthesis RCC shows the focal asymmetry as a spiculated mass (arrow).

ILC lesions on combined DM and DBT studies and the differences between the modalities in lesion conspicuity as perceived by the 2 readers. Architectural distortions (57\%) and masses (43\%) were the most frequent characteristics identified. Findings were more conspicuous on DBT than on DM studies; the sole exception was a mass lesion in a fatty breast.

Microcalcifications were identified in 4 cases. In 3 of them, there was no additional mass lesion, and the microcalcifications were equally evident on DM and DBT studies. Pathologic evaluation yielded pure ILC ( 2 cases) or combined ILC and lobular carcinoma in situ ( 1 case). In the 4 th case, which was also characterized by architectural distortion and a mass lesion, the lesion was more easily discerned on DBT than on DM studies (fig. 2). Pathologic evaluation yielded combined ILC and invasive ductal carcinoma.

Most of the masses $(7 / 10,70 \%)$ were classified by DBT as having spiculated margins. DBT changed the classification in all of these cases. The DM classification (without DBT) was a mass with indistinct margins in 4 cases, asymmetric density in 2 cases, and calcifications in 1 case. Overall, by both DM and DBT, 'star-like

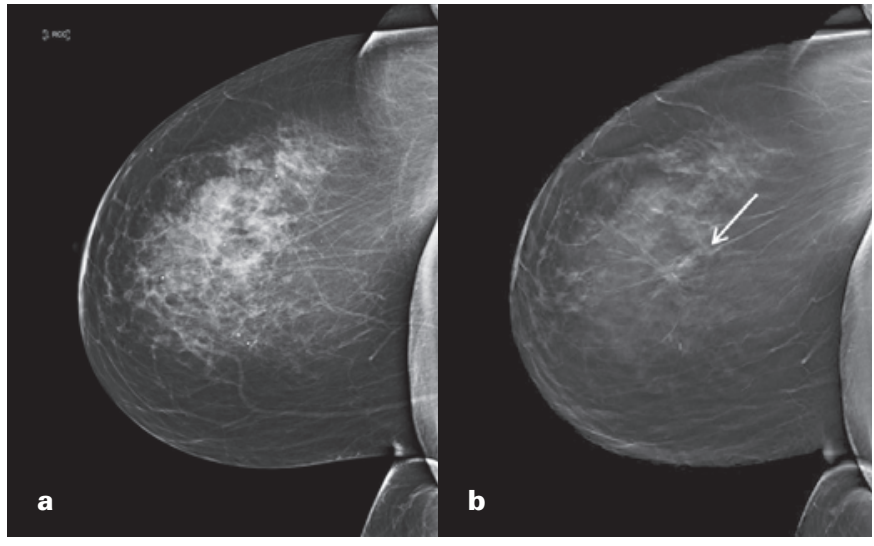

Fig. 4. 72-year-old woman, screening mammogram. a Digital mammography right craniocaudal (RCC) view - negative. b Digital breast tomosynthesis RCC demonstrates architectural distortion (arrow).

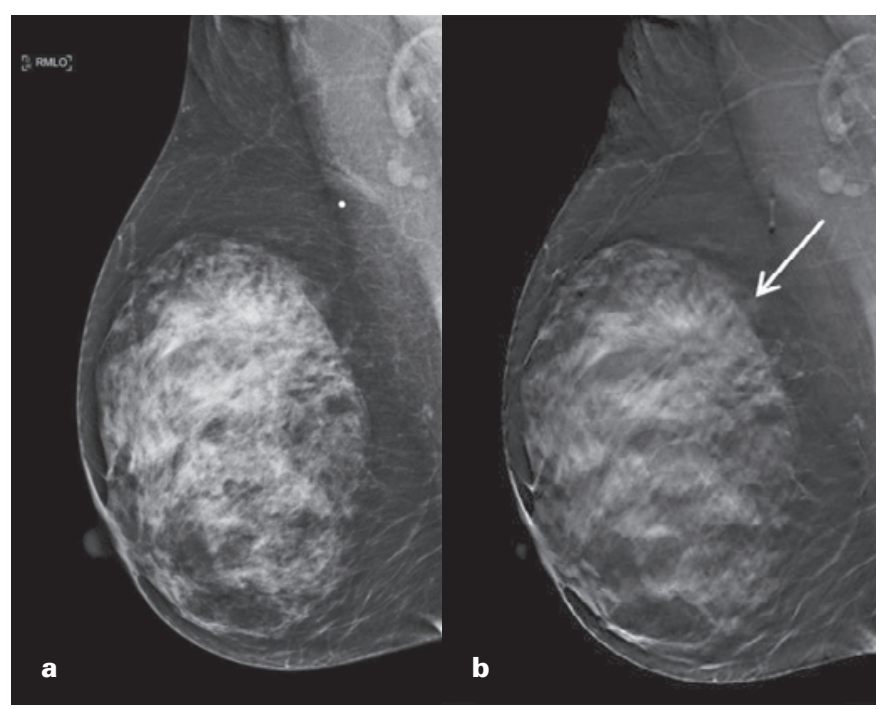

Fig. 5. 62-year-old woman, screening mammogram. a Digital mammography right mediolateral oblique (RMLO) view - BI-RADS 4 breast density, read as negative. b Digital breast tomosynthesis RMLO demonstrates architectural distortion (arrow).

lesions' (i.e., spiculated masses or architectural distortions) were documented in 20 (87\%) of the 23 cases (fig. $3-5$ ).

Two (9\%) patients had negative findings on both DM and DBT. In 1 of them, the ILC mass was located very peripherally and laterally and was therefore not included in the CC or MLO views (fig. 6). The other patient, aged 44 years, had been found to have metastatic breast carcinoma in an ovary during oophorectomy. The primary was diagnosed 


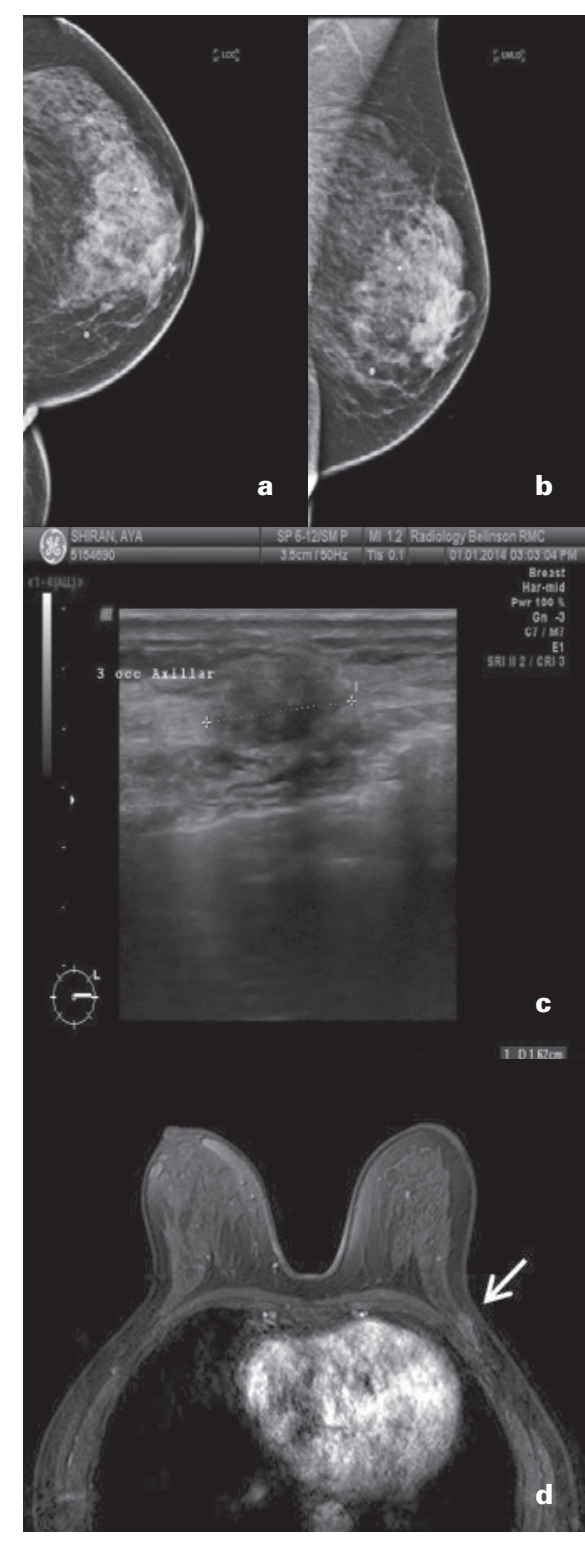

only after the ovarian operation on breast MRI which demonstrated faint asymmetric enhancements, followed by MR-directed ultrasound and core biopsy. DM and DBT in this case demonstrated BI-RADS category 4 and a dense breast pattern, and were unremarkable.

\section{Discussion}

There is well-documented evidence that the use of DBT improves cancer detection rates and reduces recall rates, at the price of slightly increased radiation doses $[5,9,10]$. The superior lesion detection achieved with the addition of DBT to DM was comparable to using additional views [6-8]. The better depiction of spiculations and architectural distortions is suggested by the reported increase in radial scar detection in screening programs using DBT [11].

The most common presentation of ILC on standard mammography is a mass, seen in $45-65 \%$ of cases. Architectural distortion is seen in $10-34 \%[2,12-14]$. In the present study, when DBT was added to DM, the rate of detection of architectural distortions rose to $57 \%$. Mass margins were better perceived, allowing ill-defined margins to be appreciated as spiculated margins. $70 \%$ of the mass lesions demonstrated spiculations.

Our study was limited by the small sample size and retrospective design. Readers were aware of the pathology, making this a descriptive work rather than a detection study (detection rates were therefore not measured). Furthermore, all patients were derived from our practice, and both readers were from our institution.

In conclusion, there is an agreement that detection of ILC on screening mammograms is particularly difficult. This study shows that the use of DBT emphasizes the ILC lesion appearance, defines lesion margins and spiculations, and highlights architectural distortion.

\section{Disclosure Statement}

The authors have no conflicts of interest.

\section{References}

1 Lopez JK, Bassett LW: Invasive lobular carcinoma of the breast: spectrum of mammographic, US, and MR imaging findings. Radiographics 2009;29:165-176

2 Krecke KN, Gisvold JJ: Invasive lobular carcinoma of the breast: mammographic findings and extent of disease at diagnosis in 184 patients. AJR Am J Roentgenol 1993; 161:957-960.

3 Tavassoli FA, Devilee P: World Health Organization Classification of Tumours, Pathology and Genetics: Tumours of the Breast and Female Genital Organs. Lyon, France, PA, IARC Press, 2003.

4 Evans WP, Warren Burhenne LJ, et al.: Invasive lobular carcinoma of the breast: mammographic characteristics and computer-aided detection. Radiology 2002; 225:182-189.

5 Svahn TM, Houssam N, Sechopoulos I, Mattsson S: Review of radiation dose estimates in digital breast tomosynthesis relative to those in two-view full-field digital mammography. Breast 2015;24:93-99.
6 Zuley ML, Bandos AI, Ganott MA, et al.: Digital breast tomosynthesis versus supplemental diagnostic mammographic views for evaluation of noncalcified breast lesions. Radiology 2013;266:89-95.

7 Gur D, Bandos AI, Rockette HE, et al.: Localized detection and classification of abnormalities on FFDM and tomosynthesis examinations rated under an FROC paradigm. AJR Am J Roentgenol 2011;196:737-741.

8 Noroozian M, Hadjiiski L, Rahnama-Moghadam S, et al.: Digital breast tomosynthesis is comparable to mammographic spot views for mass characterization. Radiology 2012;262:61-68.

9 Ciatto S, Houssami N, Bernardi D, et al.: Integration of 3D digital mammography with tomosynthesis for population breast-cancer screening (STORM): a prospective comparison study. Lancet Oncol 2013;14:583-589.
10 Haas BM, Kalra V, Geisel J, et al.: Comparison of tomosynthesis plus digital mammography and digital mammography alone for breast cancer screening. Radiology 2013;269:694-700.

11 Thomas M, Roth R, McCarthy A, Conant E: Improved conspicuity of architectural distortion with digital breast tomosynthesis increases radial sclerosing lesion detection during prospective breast cancer screening. Oral Presentation, ARRS, San Diego, 2014.

12 Hilleren DJ, Andersson IT, Lindholm K, Linnell FS: Invasive lobular carcinoma: mammographic findings in a 10-year experience. Radiology 1991;178:149-154.

13 Le Gal M, Ollivier L, Asselain B, et al.: Mammographic features of 455 invasive lobular carcinomas. Radiology 1992;185:705-708.

14 Helvie MA, Paramagul C, Oberman HA, Adler DD: Invasive lobular carcinoma: imaging features and clinical detection. Invest Radiol 1993;28:202-207. 